DOI: 10.12957/demetra.2016.22030

\title{
Estado nutricional, atividade física e tempo de tela em escolares de 7-10 anos: um estudo de intervenção em Vitória-ES
}

\section{The nutritional status, physical activity and screen time in students from 7-10 years: an intervention study in Vitória-ES, Brazil}

Lara Fernandes Coelho ${ }^{1}$

Jordana Herzog Siqueiral

Maria del Carmen Bisi Molina'

1 Universidade Federal do Espírito Santo, Departamento de Educação Integrada em Saúde. Vitória-ES, Brasil.

Financiamento: Fundação de Amparo à Pesquisa do Espírito Santo (FAPES), processo 60927089/13.

Correspondência / Correspondence Maria del Carmen Bisi Molina Universidade Federal do Espírito Santo, Departamento de Educação Integrada em Saúde Av. Marechal Campos, 1468 - Maruípe, CEP 29.040-090, Vitória-ES, Brasil.

E-mail:mdmolina@uol.com.br

\section{Resumo}

Objetivo: Avaliar a efetividade de um programa de educação em saúde sobre o estado nutricional, a prática de atividade física e o tempo de tela em escolares na faixa etária de 7 a 10 anos. Metodologia: Trata-se de um estudo de intervenção, realizado com escolares matriculados em duas escolas públicas, denominadas grupo intervenção (GI) e grupo controle (GC). A coleta de dados foi feita antes e após a intervenção usando os mesmos protocolos. Medidas antropométricas e informações sobre hábitos de vida foram coletadas nos momentos inicial e final. Na escola Intervenção foram realizadas 11 oficinas educativas versando sobre alimentação saudável e prática de atividade física. Foram utilizados testes estatísticos para avaliação das diferenças entre e intragrupos, $\mathrm{p}<0,05$ e programa SPSS 18.0. Resultados: De 215 escolares, $136(63,3 \%)$ pertenciam ao GI e $79(36,7 \%)$ ao GC; a maioria, à classe socioeconômica $\mathrm{C}$ e raça/cor não branca. Cerca de um terço dos escolares apresentavam sobrepeso ou obesidade no momento inicial. Ao final do estudo, em ambos os grupos, foi observado aumento significativo de peso, estatura, índice de massa corporal e perímetro da cintura, embora o percentual de excesso de peso não tenha aumentado. Houve redução significativa do tempo de tela no GI $(\mathrm{p}<0,023)$ após intervenção. Conclusão: As alterações das medidas antropométricas são esperadas devido ao momento em que os escolares se encontram, porém o programa de educação em saúde impactou positivamente na prevenção de novos casos de excesso de peso e na redução do tempo de tela.

Palavras-chave: Criança. Estudos de Intervenção. Atividade Motora. Sobrepeso. 


\section{Abstract}

Objective: Evaluating the effectiveness of a health education program on the nutritional status, level of physical activity and screen time in students aged 7-10 years. Methodology: This is an intervention study which was conducted with 215 children enrolled in two public schools, hereinafter called the intervention group (IG) and control group (CG). The data collection was performed before and after the intervention using the same protocols. The anthropometric measurements and information about lifestyle habits were collected in two schools in the beginning and ending of school period. At the Intervention school, eleven educational workshops were held on healthy eating and physical activity practice. Statistical tests were used to evaluate the differences between and within groups, $\mathrm{p}<0.05$ and SPSS 18.0 program. Results: From all 215 students, 136 (63.3\%) belonged to GI and 79 (36.7\%) to the GC; most of them belonged to the socioeconomic class $\mathrm{C}$ and not white race/color. About a third of the students were overweight or obese at the initial time. At the end of the study, in both groups, there was a significant increase in weight, height, body mass index and waist circumference, although the overweight percentage has not increased. There was a significant reduction in screen time in GI $(\mathrm{p}<0.023)$ after intervention. Conclusion: Changes in the anthropometric measures are expected due to the moment in which children are, but health education program had a positive impact in preventing new cases of overweight and reducing the screen time.

Key words: Child. Intervention Studies. Motor Activity. Overweight.

\section{Introdução}

O sobrepeso e a obesidade atingem todas as faixas etárias e são considerados problema de saúde pública, tanto em países de renda alta como nos de média e baixa renda. ${ }^{1}$ São desfechos de etiologia multifatorial e associados a fatores biológicos, psicológicos, socioeconômicos e/ou socioambientais. ${ }^{2}$ Além disso, está bem documentado na literatura o fato de o excesso de peso representar um importante fator de risco para as Doenças Crônicas Não Transmissíveis (DCNTs).1 Dessa forma, atenção especial é necessária ao excesso de peso infantil, uma vez que é um fator agravante para a manutenção do estado nutricional inadequado na vida adulta. ${ }^{3}$

Cerca de um terço das crianças brasileiras de 5 a 9 anos $(33,5 \%)$ encontra-se com excesso de peso e $14,3 \%$, com obesidade, e na faixa etária de 10 a 11 anos, $28,6 \%$ e 8,6\%, respectivamente. ${ }^{4}$ 
Paralelamente, estudos mostram que crianças têm dedicado tempo em excesso para atividades de lazer sedentário, ${ }^{5,6}$ ultrapassando o limite de duas horas diárias, proposto pela Academia Americana de Pediatria (AAP), ${ }^{7}$ com dispositivos de tela (televisão, vídeo game e computador). Em contrapartida, a prática de atividade física da população mais jovem sofre uma tendência de redução. ${ }^{8,9}$

Partindo da perspectiva de que o estado nutricional ${ }^{3}$ e hábitos de atividade física ${ }^{10}$ tendem a perdurar em faixas etárias posteriores, a infância parece ser o momento ideal para promoção de hábitos saudáveis e as escolas se mostram como um ambiente propício para a execução de intervenções voltadas para a saúde. ${ }^{11,12}$

Embora de grande relevância, há poucos estudos de intervenção entre escolares no Brasil. Assim sendo, o objetivo do presente trabalho foi avaliar a efetividade de um programa de saúde sobre o estado nutricional, a atividade física e o tempo de tela em escolares de Vitória/ES.

\section{Metodologia}

Trata-se de um estudo de intervenção desenvolvido em duas escolas da rede pública de ensino de Vitória-ES, no ano de 2014. Este estudo faz parte de um projeto intitulado "Práticas saudáveis de alimentação e nutrição em ambiente escolar: Construção, desenvolvimento e avaliação".

De acordo com os dados da Secretaria Municipal de Educação de Vitória, a região de Saúde Centro contemplava cinco Escolas Municipais de Ensino Fundamental (EMEF) com escolares na faixa etária dos 7 aos 10 anos de idade, totalizando 450 estudantes. A amostra foi constituída por 215 escolares nessa mesma faixa etária e região. Das cinco escolas da região, foram sorteadas duas e, em seguida, foi realizado outro sorteio para identificação da escola que receberia o Programa de Intervenção em Saúde. Essa escola foi denominada Grupo Intervenção (GI) e a outra escola, Grupo Controle (GC).

Todos os escolares do $2^{\underline{\alpha}}$ ao $5^{\mathrm{o}}$ ano foram convidados a participar da pesquisa, sendo que os participantes foram incluídos no estudo mediante assinatura do Termo de Consentimento Livre e Esclarecido pelos pais/responsáveis, e do Termo de Assentimento, pelo escolar.

Obedecendo aos aspectos éticos, esta investigação foi aprovada pelo Comitê de Ética em Pesquisa do Centro de Ciências da Saúde da Universidade Federal do Espírito Santo sob parecer de número 004061/2013, está registrada na Organização Mundial de Saúde sob o UTN - U111111553103 e submetida ao Registro Brasileiro de Ensaios Clínicos sob o número REQ: 2461, em 2/4/2014.

A coleta de dados foi realizada no ambiente escolar em dois momentos, no início e ao final da intervenção, por estudantes dos cursos de graduação em Nutrição e Educação Física da Universidade Federal do Espírito Santo, devidamente treinados para este fim. 
O peso foi aferido em balança eletrônica da marca Tanita ${ }^{\circledR}$ com capacidade máxima para 150

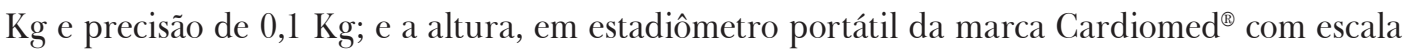
de $0,1 \mathrm{~cm}$. Ambas as medidas foram realizadas com o mínimo de roupas possível, descalças e livres de adornos na cabeça. O índice de massa corporal (IMC) foi calculado por meio da razão entre a massa corporal $(\mathrm{Kg})$ e o quadrado da estatura $\left(\mathrm{m}^{2}\right)$. Aferiu-se a medida do perímetro da cintura (PC) com o escolar em pé, braços estendidos ao longo do corpo no ponto médio entre a margem inferior da última costela e a crista ilíaca ${ }^{13}$ no plano horizontal com auxílio de fita métrica inextensível da marca Sanny Medical ${ }^{\circledR}$.

Foi aplicado um questionário estruturado aos escolares que abordava questões de hábitos de vida. Também foi enviado um questionário ao domicílio para preenchimento pelos pais/responsáveis com perguntas sobre hábitos de vida da família e do escolar e sobre lazer sedentário. Utilizou-se questionário validado por Fernandes ${ }^{14}$ a respeito do lazer sedentário.

Foram realizadas 11 atividades educativas durante o ano letivo de 2014 sobre diferentes temas, as quais estão apresentadas no Quadro 1, junto às metodologias utilizadas e objetivos. A intervenção foi baseada de acordo com diversos contextos, incluindo os contextos social, institucional e cultural, partindo da perspectiva de que os problemas de saúde são influenciados por fatores individuais e ambientais. ${ }^{15}$

Quadro 1. Temas, metodologia e objetivos das atividades de intervenção. Vitória-ES, 2014.

\begin{tabular}{|c|c|c|}
\hline Temas & Metodologias & Objetivos \\
\hline $\begin{array}{c}\text { Preferências } \\
\text { alimentares e relação com a } \\
\text { alimentação }\end{array}$ & $\begin{array}{l}\text { Registro com palavras ou } \\
\text { desenhos dos alimentos que } \\
\text { agradam, desagradam e } \\
\text { fazem bem à saúde }\end{array}$ & $\begin{array}{c}\text { Conhecer os hábitos } \\
\text { alimentares dos escolares e } \\
\text { compreender o processo de } \\
\text { alimentação }\end{array}$ \\
\hline $\begin{array}{l}\text { Identificação dos locais } \\
\text { de venda de alimentos } \\
\text { próximos à escola e à } \\
\text { residência }\end{array}$ & $\begin{array}{l}\text { Observação dos arredores } \\
\text { da escola e de casa } \\
\text { com registro do que foi } \\
\text { observado }\end{array}$ & $\begin{array}{l}\text { Conhecer locais de venda de } \\
\text { alimentos na vizinhança da } \\
\text { criança e da escola }\end{array}$ \\
\hline $\begin{array}{c}\text { Diversidade de frutas e } \\
\text { hortaliças e o benefício } \\
\text { das cinco cores (vermelha, } \\
\text { laranja, verde, roxa e } \\
\text { branca) }\end{array}$ & $\begin{array}{c}\text { Dinâmica com charadas } \\
\text { relacionadas a frutas e } \\
\text { hortaliças }\end{array}$ & $\begin{array}{l}\text { Abordar e mostrar a } \\
\text { importância - por meio } \\
\text { de jogos interativos - } \\
\text { dos benefícios de uma } \\
\text { alimentação colorida }\end{array}$ \\
\hline
\end{tabular}




\begin{tabular}{|c|c|c|}
\hline Temas & Metodologias & Objetivos \\
\hline $\begin{array}{c}\text { Rotulagem de alimentos e } \\
\text { propagandas televisivas }\end{array}$ & $\begin{array}{l}\text { Leitura dos rótulos de } \\
\text { alimentos e exibição de } \\
\text { propagandas bem como } \\
\text { de tubos de ensaio com } \\
\text { quantidades de sal, açúcar } \\
\text { e gordura contidas em } \\
\text { alimentos consumidos pelo } \\
\text { público infantil }\end{array}$ & $\begin{array}{c}\text { Despertar a curiosidade } \\
\text { para leitura dos rótulos } \\
\text { e alertar sobre as } \\
\text { propagandas de alimentos, } \\
\text { bem como desenvolver o } \\
\text { senso crítico }\end{array}$ \\
\hline Brincadeiras ativas & $\begin{array}{l}\text { Circuito de brincadeiras, } \\
\text { (amarelinha, elástico e } \\
\text { corrida com balões) e } \\
\text { resgate às brincadeiras dos } \\
\text { pais por meio de roda de } \\
\text { conversa }\end{array}$ & $\begin{array}{c}\text { Incentivar a prática de } \\
\text { atividade física e mostrar } \\
\text { que não é necessário muito } \\
\text { espaço ou recursos para se } \\
\text { movimentar }\end{array}$ \\
\hline $\begin{array}{c}\text { Revisão dos benefícios das } \\
\text { cinco cores de frutas e } \\
\text { hortaliças }\end{array}$ & $\begin{array}{l}\text { Dinâmica de preenchimento } \\
\text { de cartazes com nomes de } \\
\text { frutas e hortaliças de cada } \\
\text { uma das cinco cores }\end{array}$ & $\begin{array}{l}\text { Enfatizar novamente os } \\
\text { benefícios do consumo } \\
\text { regular de frutas e } \\
\text { hortaliças, por meio de } \\
\text { análise de seus nutrientes a } \\
\text { partir das cores }\end{array}$ \\
\hline Degustação de frutas & $\begin{array}{l}\text { Dinâmica de degustação } \\
\text { às cegas para adivinhação, } \\
\text { na qual os escolares } \\
\text { experimentaram frutas do } \\
\text { seu cotidiano, bem como } \\
\text { frutas exóticas }\end{array}$ & $\begin{array}{l}\text { Mostrar a diversidade } \\
\text { de frutas e incentivar o } \\
\text { consumo regular }\end{array}$ \\
\hline Avaliação das intervenções & $\begin{array}{l}\text { Registro com palavras ou } \\
\text { desenhos das preferências } \\
\text { alimentares, das mudanças } \\
\text { ocorridas a partir das } \\
\text { oficinas e dos alimentos que } \\
\text { pretendem experimentar }\end{array}$ & $\begin{array}{c}\text { Observar as mudanças/efeito } \\
\text { das oficinas }\end{array}$ \\
\hline
\end{tabular}




\begin{tabular}{|c|c|c|}
\hline Temas & Metodologias & Objetivos \\
\hline $\begin{array}{c}\text { Identificação das } \\
\text { companhias para } \\
\text { brincadeiras e atividade } \\
\text { física }\end{array}$ & $\begin{array}{c}\text { Divisão de grupos para } \\
\text { atividade de corrida e } \\
\text { perguntas sobre afetividade }\end{array}$ & $\begin{array}{c}\text { Coletar relatos de } \\
\text { afetividade e prática de } \\
\text { atividade física fora da } \\
\text { escola }\end{array}$ \\
\hline $\begin{array}{c}\text { Identificação de locais para } \\
\text { prática de atividade física }\end{array}$ & $\begin{array}{c}\text { Divisão de grupos } \\
\text { para registro dos } \\
\text { locais disponíveis para } \\
\text { brincadeiras e prática de } \\
\text { atividade física próximos } \\
\text { das residências }\end{array}$ & $\begin{array}{c}\text { Conhecer os locais próximos } \\
\text { a suas residências que }\end{array}$ \\
& $\begin{array}{c}\text { para brincar } \\
\text { Revisão das oficinas } \\
\text { de material didático para } \\
\text { leitura com a família }\end{array}$ & $\begin{array}{c}\text { Relembrar e fixar os } \\
\text { conteúdos das oficinas }\end{array}$ \\
\hline
\end{tabular}

Imprescindivelmente, no início ou ao final de todas as oficinas educativas, foram feitas atividades de respiração, alongamento e aquecimento utilizando brincadeiras com o intuito de promover ou despertar o interesse pelo hábito da prática de atividade física. Os escolares do GC também receberam informações relativas aos temas das oficinas ao final do estudo.

A raça/cor dos escolares foi classificada pelos entrevistadores e a condição socioeconômica familiar de acordo com o Critério de Classificação Econômica Brasil (ABEP). ${ }^{16}$

Na análise do IMC para idade foram utilizadas as curvas da OMS 2006-2007 de acordo com o escore z de baixo peso (escore $\mathrm{z}<-1$ ), eutrofia (escore $\mathrm{z}-1 \mathrm{a}+1$ ) e excesso de peso (escore $\mathrm{z}>$ $+1) .{ }^{17}$ Para a classificação do PC foram adotados os pontos de corte propostos por Taylor et al. ${ }^{18}$ Os escolares com percentil acima de 90 foram classificados com obesidade abdominal.

O tempo de tela foi analisado de acordo com o somatório do tempo diário gasto com dispositivos de tela (televisão, vídeo game e computador). Para essa variável foi utilizado o ponto de corte igual a 120 minutos, tempo máximo recomendado pela Academia Americana de Pediatria (AAP), ${ }^{7}$ e 
os escolares foram classificados como "atende" o limite de 120 minutos (aqueles que gastam 120 minutos ou menos diariamente com dispositivos de tela) e "não atende" o limite de 120 minutos (aqueles que gastam mais que 120 minutos diariamente com dispositivos de tela).

A prática de atividade física foi quantificada de acordo com o somatório do tempo de atividade física supervisionada, brincadeiras ativas (futebol, corrida, dança, natação, ciclismo, piques) e deslocamento ativo casa-escola-casa. Para essa variável foi utilizado o ponto de corte igual a 300 minutos semanais, considerando o valor mínimo de 60 minutos diários recomendado para crianças. ${ }^{19}$ Desta forma, os escolares foram classificados como "ativos" (aqueles que somaram 300 minutos ou mais) e "insuficientemente ativos" (aqueles que somaram menos de 300 minutos). Ressalta- se que as informações a respeito do lazer sedentário dos escolares foram computadas pelos pais/responsáveis por meio de preenchimento do questionário.

Para descrever as variáveis de estudo, utilizou-se medidas de tendência central e medidas de dispersão para as variáveis contínuas, e percentuais para as variáveis categóricas. Foi realizado teste de Kolmogorov Smirnov para testar a normalidade das variáveis e as estatísticas foram realizadas de acordo com esse resultado. Para a análise das diferenças das proporções em variáveis qualitativas foi utilizado o teste do qui-quadrado, Teste Exato de Fisher e McNemar. Nas variáveis contínuas, utilizou-se o teste de Mann-Whitney para verificar diferenças entre amostras independentes e teste de Wilcoxon para verificar diferenças entre amostras pareadas. O nível de significância estatística adotado foi $\mathrm{p}<0,05$. As análises foram realizadas através do programa estatístico SPSS versão 18.0.

\section{Resultados}

A amostra deste estudo foi composta por 215 escolares, dos quais $136(63,3 \%)$ pertenciam ao GI e $79(36,7 \%)$ ao GC, 123 (57,2\%) eram do sexo feminino, 92 (42,8\%) do sexo masculino e mais de $90 \%$ (n=196) moravam com a mãe na avaliação inicial. Observou-se diferença estatística entre os grupos de alocação quanto à raça/cor, classe socioeconômica e escolaridade materna. No GC, cerca de $80 \%(n=65)$ eram de não brancos (pardos e negros), 75\%(n=59) pertenciam à classe socioeconômica $\mathrm{C}$ e

$39 \%$ ( $\mathrm{n}=31)$ das mães possuíam Ensino Médio completo, Superior completo e Pós- graduação. Já no GI, 42,6\% (n=58) eram brancos, 44,9\% (n=61) das classes socioeconômicas A e B e 66\% $(\mathrm{n}=90)$ das mães possuíam Ensino Médio completo, Superior completo e Pós-graduação, como pode ser observado na Tabela 1. 
Tabela 1. Caracterização da amostra segundo grupo de alocação na avaliação inicial. VitóriaES, 2014.

\begin{tabular}{|c|c|c|c|c|c|c|c|}
\hline \multirow[t]{2}{*}{ Variáveis } & \multicolumn{2}{|c|}{$\begin{array}{c}\text { Grupo } \\
\text { Intervenção }\end{array}$} & \multicolumn{2}{|c|}{$\begin{array}{l}\text { Grupo } \\
\text { Controle }\end{array}$} & \multirow[t]{2}{*}{$\begin{array}{l}\text { Valor } \\
\text { de p }\end{array}$} & \multicolumn{2}{|c|}{ Total } \\
\hline & $\mathbf{n}$ & $\%$ & $\mathbf{n}$ & $\%$ & & $\mathrm{n}$ & $\%$ \\
\hline Sexo & & & & & 0,955 & & \\
\hline Masculino & 58 & 42,6 & 34 & 43,0 & & 92 & 42,8 \\
\hline Feminino & 78 & 57,4 & 45 & 57,0 & & 123 & 57,2 \\
\hline Idade (anos) & & & & & 0,276 & & \\
\hline 7 & 37 & 27,2 & 22 & 27,8 & & 59 & 27,4 \\
\hline 8 & 31 & 22,8 & 16 & 20,3 & & 47 & 21,9 \\
\hline 9 & 27 & 19,9 & 24 & 30,4 & & 51 & 23,7 \\
\hline 10 & 41 & 30,1 & 17 & 21,5 & & 58 & 27,0 \\
\hline Raça/cor & & & & & $<0,001$ & & \\
\hline Branco & 58 & 42,6 & 14 & 17,7 & & 72 & 33,5 \\
\hline Não branco & 78 & 57,4 & 65 & 82,3 & & 143 & 66,5 \\
\hline Classe socioeconômica & & & & & $<0,001$ & & \\
\hline $\mathrm{A}+\mathrm{B}$ & 61 & 44,9 & 11 & 13,9 & & 72 & 33,5 \\
\hline $\mathrm{C}$ & 62 & 45,6 & 59 & 74,7 & & 121 & 56,3 \\
\hline $\mathrm{D}+\mathrm{E}$ & 13 & 9,6 & 9 & 11,4 & & 22 & 10,2 \\
\hline Mora com a mãe & & & & & 0,993 & & \\
\hline Sim & 124 & 91,2 & 72 & 91,1 & & 196 & 91,2 \\
\hline Não & 12 & 8,8 & 7 & 8,9 & & 19 & 8,8 \\
\hline Escolaridade materna $^{* *}$ & & & & & $<0,001$ & & \\
\hline $\begin{array}{l}\text { Analfabeto/Fundamental } \\
\text { incompleto }\end{array}$ & 24 & 17,6 & 30 & 38,0 & & 54 & 25,1 \\
\hline Fundamental completo & 22 & 16,2 & 18 & 22,8 & & 40 & 18,6 \\
\hline $\begin{array}{l}\text { Médio completo/Superior } \\
\text { Completo/Pós-Graduação }\end{array}$ & 90 & 66,2 & 31 & 39,2 & & 121 & 56,3 \\
\hline
\end{tabular}

Teste qui-quadrado.**Teste Exato de Fisher. $\mathrm{n}=215$. 
A Tabela 2 mostra que não houve diferença estatisticamente significante quanto aos hábitos de vida e medidas antropométricas entre os dois grupos na linha de base. Observa-se que a maioria dos escolares de ambos os grupos eram eutróficos, apresentavam PC adequado e classificados como ativos, entretanto, não atendiam à recomendação de tempo de tela diário. Não foi verificada diferença estatística intragrupo em relação às variáveis de estado nutricional e hábitos de vida antes e após as intervenções (Tabela 3).

Tabela 2. Variáveis antropométricas e de hábitos de vida na avaliação inicial segundo grupo de alocação. Vitória-ES, 2014.

\begin{tabular}{|c|c|c|c|c|c|}
\hline \multirow[t]{3}{*}{ Variáveis } & \multicolumn{4}{|c|}{ Avaliação inicial } & \multirow{3}{*}{ Valor de $\mathrm{p}$} \\
\hline & \multicolumn{2}{|c|}{ GI } & \multicolumn{2}{|c|}{ GC } & \\
\hline & $\mathrm{n}$ & $\%$ & $\mathbf{n}$ & $\%$ & \\
\hline IMC & & & & & 0,082 \\
\hline Eutrofia & 84 & 61,8 & 58 & 73,4 & \\
\hline Excesso de peso & 52 & 38,2 & 21 & 26,6 & \\
\hline PC & & & & & 0,053 \\
\hline Adequado & 84 & 61,8 & 59 & 74,7 & \\
\hline Obesidade abdominal & 52 & 38,2 & 20 & 25,3 & \\
\hline Tempo total de AtF & & & & & 0,622 \\
\hline$\geq 300 \mathrm{~min} / \mathrm{sem}$ & 117 & 86,0 & 66 & 83,5 & \\
\hline$<300 \mathrm{~min} / \mathrm{sem}$ & 19 & 14,0 & 13 & 16,5 & \\
\hline Tempo de tela & & & & & 0,530 \\
\hline$\leq 120 \mathrm{~min} / \mathrm{dia}$ & 15 & 11,0 & 11 & 13,9 & \\
\hline$>120 \mathrm{~min} / \mathrm{dia}$ & 121 & 89,0 & 68 & 86,1 & \\
\hline
\end{tabular}

Teste qui-quadrado. GI = Grupo Intervenção. GC $=$ Grupo Controle. IMC $=$ Índice de Massa Corporal. $\mathrm{PC}=$ Perímetro da Cintura. AtF $=$ Atividade Física. $\mathrm{n}=215$. 
Tabela 3. Variáveis antropométricas e de hábitos de vida segundo grupo de alocação. Vitória-ES, 2014.

\begin{tabular}{|c|c|c|c|c|c|c|c|c|c|c|}
\hline \multirow{3}{*}{ Variáveis } & \multicolumn{4}{|c|}{ Grupo Intervenção* } & \multirow{3}{*}{$\begin{array}{l}\text { Valor } \\
\text { de p }\end{array}$} & \multicolumn{4}{|c|}{ Grupo Controle** } & \multirow{3}{*}{$\begin{array}{l}\text { Valor } \\
\text { de p }\end{array}$} \\
\hline & \multicolumn{2}{|c|}{ AI } & \multicolumn{2}{|c|}{$\mathrm{AF}$} & & \multicolumn{2}{|c|}{ AI } & \multicolumn{2}{|c|}{$\mathrm{AF}$} & \\
\hline & $\mathrm{n}$ & $\%$ & $\mathrm{n}$ & $\%$ & & $\mathrm{n}$ & $\%$ & $\mathrm{n}$ & $\%$ & \\
\hline IMC & & & & & 0,289 & & & & & 0,687 \\
\hline Eutrofia & 84 & 61,8 & 80 & 58,8 & & 58 & 73,4 & 60 & 75,9 & \\
\hline Excesso de peso & 52 & 38,2 & 56 & 41,2 & & 21 & 26,6 & 19 & 24,1 & \\
\hline PG & & & & & 1,000 & & & & & 0,687 \\
\hline Adequado & 84 & 61,8 & 84 & 61,8 & & 59 & 74,7 & 61 & 77,2 & \\
\hline $\begin{array}{l}\text { Obesidade } \\
\text { abdominal }\end{array}$ & 52 & 38,2 & 52 & 38,2 & & 20 & 25,3 & 18 & 22,8 & \\
\hline Tempo total de & & & & & 0,227 & & & & & 1,000 \\
\hline \multicolumn{11}{|l|}{ AtF } \\
\hline$\geq 300 \mathrm{~min} / \mathrm{sem}$ & 117 & 86,0 & 122 & 89,7 & & 66 & 83,5 & 66 & 83,5 & \\
\hline$<300 \mathrm{~min} / \mathrm{sem}$ & 19 & 14,0 & 14 & 10,3 & & 13 & 16,5 & 13 & 16,5 & \\
\hline Tempo de tela & & & & & 0,607 & & & & & 0,453 \\
\hline$\leq 120 \mathrm{~min} / \mathrm{dia}$ & 15 & 11,0 & 18 & 13,2 & & 11 & 13,9 & 14 & 17,7 & \\
\hline$>120 \mathrm{~min} / \mathrm{dia}$ & 121 & 89,0 & 118 & 86,8 & & 68 & 86,1 & 65 & 82,3 & \\
\hline
\end{tabular}

Teste McNemar. AI = Avaliação inicial. AF = Avaliação final. AtF = Atividade física. IMC = Índice de Massa Corporal. $\mathrm{PC}=$ Perímetro da Cintura. $\mathrm{n}=215 . * \mathrm{n}=136 . * * \mathrm{n}=79$.

A Tabela 4 apresenta as médias das variáveis antropométricas e de hábitos de vida nos dois momentos de avaliação por grupo de alocação. Houve diferença entre os grupos tanto na avaliação inicial e final em relação ao peso $(p=0,033$ e $p=0,030$, respectivamente), $P C(p=0,007$ e $p=0,028$, respectivamente) e ao IMC na avaliação final $(p=0,024)$. Com relação ao tempo de tela, a média de ambos os grupos foi cerca de duas vezes maior que a recomendação nos dois momentos. A média da prática de atividade física também foi superior à recomendação, cerca de três vezes.

Em relação às médias das variáveis antropométricas na avaliação inicial e final intragrupo (GI e GC) foi observada diferença estatística em relação ao peso $(\mathrm{p}<0,001)$, estatura $(\mathrm{p}<0,001)$, IMC ( $\mathrm{p}<0,001)$ e PC $(\mathrm{p}<0,001)$, com acréscimo em todas as medidas. Quanto aos hábitos de vida, observa-se que houve redução significativa do tempo de tela no GI $(\mathrm{p}<0,023)$ após a intervenção, como mostra a Tabela 5 . 


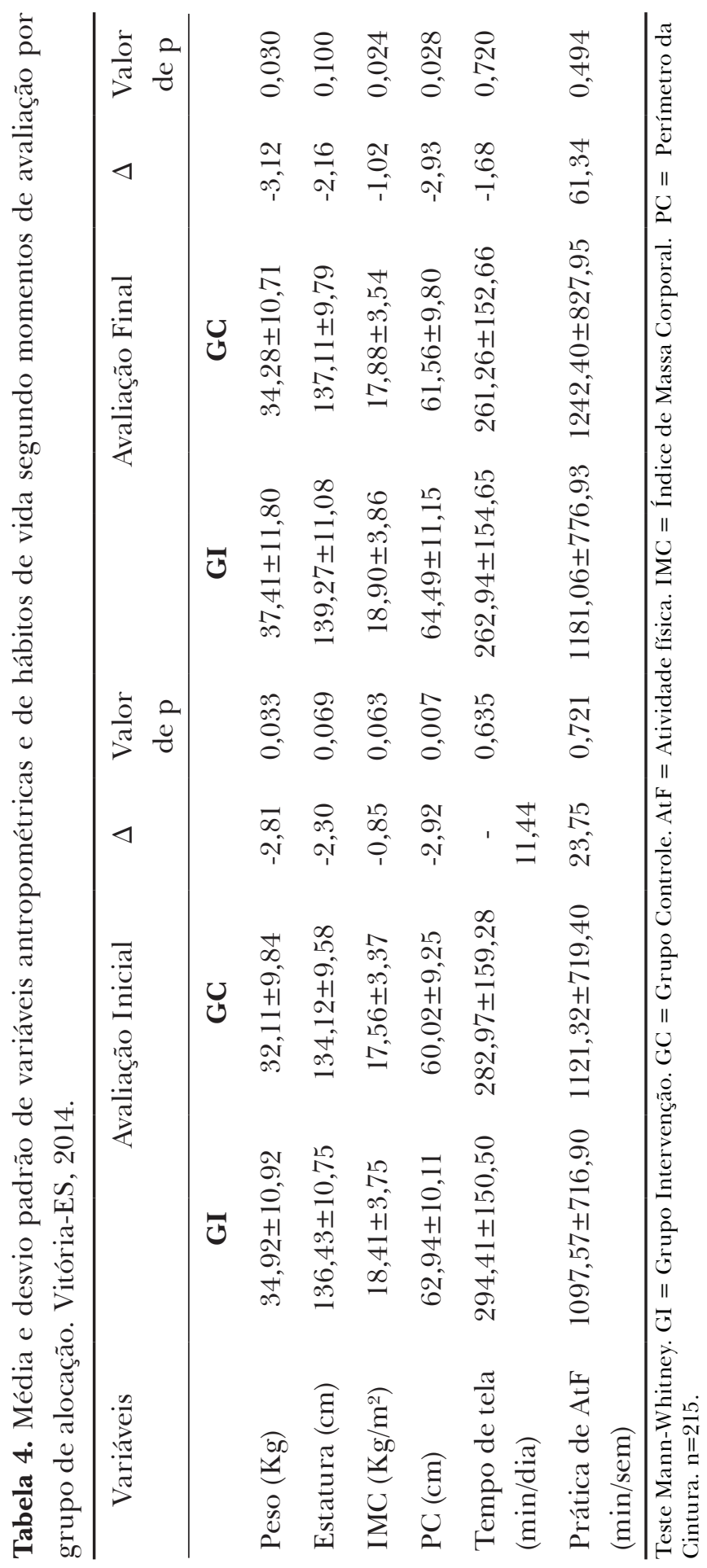




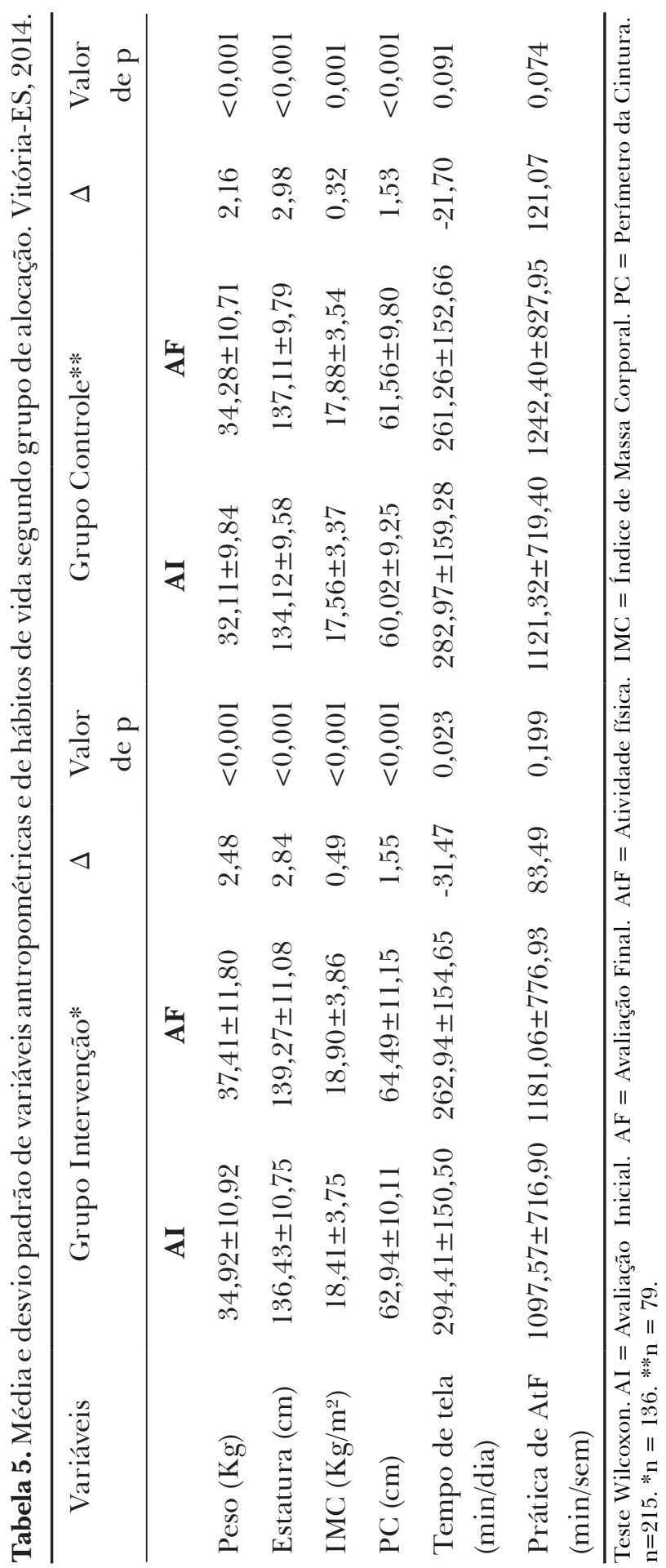




\section{Discussão}

Os resultados obtidos neste estudo apontam para a efetividade do programa de intervenção em saúde na redução do lazer sedentário em escolares de 7 a 10 anos, uma vez que foi observada redução estatisticamente significativa no tempo de tela apenas no GI após a intervenção.

Em relação ao estado nutricional da amostra, no início do estudo, cerca de um terço encontravase com sobrepeso/obesidade, o que representa resultado mediano ao considerar os valores encontrados em outras cidades brasileiras. Em Campinas/SP, Castilho et al. ${ }^{20}$ encontraram 43,5\% de excesso de peso na faixa etária de 7 a 10 anos, enquanto em Maringá/PR, a prevalência foi de 24,0\%. ${ }^{21}$ No Nordeste, estado de Pernambuco, a prevalência foi de 13,3\%, incluindo crianças e adolescentes, ${ }^{22}$ e no Norte brasileiro (Belém/Pará), 20,4\% foram classificados com excesso de peso. ${ }^{23}$

O excesso de peso foi maior nos escolares do GI (38,2\%), que por sua vez também se encontravam na classe socioeconômica mais elevada. Esse resultado corrobora as evidências de que maior renda e melhor condição social estão associadas à maior prevalência de excesso de peso, como encontrado por Brasil et al. ${ }^{24}$ e Rosaneli et al. ${ }^{21}$. Contudo, a ocorrência de 26,6\% de excesso de peso no GC e a informação que essas crianças estão em extratos sociais mais baixos não deve ser ignorada, pois também se apresenta elevada. Nesse caso, pressupõe-se que a baixa escolaridade materna, o menor acesso a informações adequadas e o baixo poder aquisitivo influenciam negativamente na alimentação das crianças, assim como foi observado por Molina et al. ${ }^{25}$.

A faixa etária escolar analisada no presente estudo é caracterizada por crescimento lento, porém constante, ${ }^{26}$ ou seja, o aumento da estatura e do peso, inclusive relacionado ao aumento do tecido adiposo, é característico nessa etapa da vida, uma vez que há preparação para o estirão da puberdade. Sendo assim, o acréscimo nas variáveis antropométricas encontradas no momento final é justificado pelo desenvolvimento natural dessa fase, e o IMC, parâmetro utilizado para a classificação do estado nutricional, é proporcional ao aumento do peso e estatura. Dessa forma, os resultados significativos de aumento de peso, estatura, IMC e PC eram esperados no decorrer do ano letivo.

A elevada atividade física praticada pela amostra tanto antes quanto após as intervenções se contrapõe à tendência de redução observada entre os mais jovens como observado em Ouro Preto/ $\mathrm{MG}$, em que $80,3 \%$ das crianças e adolescentes foram classificados como inativos. ${ }^{27} \mathrm{O}$ resultado do presente estudo se deve ao local de moradia dos escolares, uma vez que a região Centro de Vitória/ES possui praças, parques, quadras e é cercada por morros e ruas de difícil acesso com automóveis, logo essas características propiciam fácil acesso a brincadeiras e o deslocamento ativo. Outro fato é a participação em projetos sociais e nas próprias escolas da região, os quais fornecem aulas supervisionadas de ginástica rítmica, dança e lutas por longos períodos do turno matutino ou vespertino. 
O elevado tempo dedicado às atividades de lazer sedentário foi uma realidade no presente estudo e corrobora os resultados de Molina et al. ${ }^{5}$ e Andrade Neto et al. ${ }^{6}$ ao estudarem crianças do mesmo município. Paralelamente, em Ouro Preto/MG, 88,4\% dos escolares despendiam mais que 2 horas com dispositivos de tela e a média de exposição diária foi de 3 horas e 30 minutos, ${ }^{27}$ tempo semelhante ao encontrado em Niterói/RJ. onde foram estudados escolares de $10 \mathrm{a} 18$ anos. ${ }^{28}$ Em São Luís/MA, a média dos alunos do $5^{\underline{0}}$ ao 70 ano foi 2 horas e 40 minutos diárias. ${ }^{29}$

A coexistência de tempo de tela e prática de atividade física altos nos dois grupos mostra que tais comportamentos não devem ser considerados opostos ou competitivos e diversos estudos apontam para o mesmo caminho. Uma meta-análise desenvolvida por Pearson et al. ${ }^{30}$ indicou que, apesar do tempo de tela e atividade física apresentarem associação inversa, a relação encontrada é fraca. Ou seja, é possível que a criança seja muito ativa e ainda assim dedique tempo em excesso ao lazer sedentário.

O programa de saúde foi efetivo para a redução do lazer sedentário e manutenção da prática de atividade física, sendo observada diferença significativa no tempo de exposição a dispositivos de tela somente no GI após a intervenção. Na Nova Zelândia, Maddison et al ${ }^{31}$ não compartilharam do mesmo achado com crianças de 9 a 12 anos, em que não foi observada diferença estatística sobre o tempo de tela e IMC ao final do estudo. Em Portugal, Lopes et al..$^{32}$ desenvolveram um programa de intervenção para a faixa etária de 6 a 12 anos com o objetivo de avaliar mudanças na prática de atividade física por meio da inserção de jogos e brincadeiras ativas no recreio escolar, sendo que o resultado foi o aumento do tempo dispendido em atividade física total para ambos os sexos e todas as faixas etárias incluídas na análise.

No panorama nacional, em Belo Horizonte/MG, Ribeiro \& Alves ${ }^{33}$ encontraram resultado positivo a partir de estudo comparativo de estratégias de intervenção, uma vez que houve redução do tempo gasto em atividades sedentárias. Costa et al. ${ }^{34}$ realizaram palestras, teatros de fantoches, dinâmicas de grupo e arte culinária como métodos de intervenção em crianças de uma escola particular de Araçatuba/SP, e não houve alteração no PC e no IMC dos escolares. Em Florianópolis/SC, Gabriel et al. ${ }^{11}$ utilizaram a Educação Alimentar e Nutricional como único método de intervenção em crianças da $3^{\underline{a}}$ e $4^{\underline{a}}$ série, sendo que não encontraram alteração no estado nutricional.

A redução do IMC e PC raramente é verificada nesses estudos. No entanto, assim como na presente análise, novos casos de sobrepeso e obesidade podem ser prevenidos pelos programas de intervenção. Ressalta-se que no GI era esperada maior incidência de excesso de peso devido à melhor condição socioeconômica e esse resultado não foi observado. Também não foi notada redução no percentual de escolares eutróficos mesmo com a crescente prevalência de obesidade entre os mais jovens no Brasil. 
No presente estudo, utilizou-se questionário validado para avaliar hábitos de vida e os pais/ responsáveis foram orientados por escrito em relação ao preenchimento do mesmo, cuidado este que poderia reduzir o viés de informação.

Uma limitação do estudo refere-se ao tempo de tela não contabilizar o tempo gasto com smartphones, uma vez que atualmente seu uso é amplo, inclusive por crianças e adolescentes.

\section{Conclusão}

O sobrepeso e a obesidade são uma realidade no público infantil e encontra-se na escola a oportunidade do desenvolvimento de ações que podem contribuir para a melhoria da saúde em geral. Propostas que incluem redução do excesso de peso, aumento da atividade física e redução de comportamentos sedentários são frequentes, mas nem sempre os três temas são abordados de forma simultânea, como realizado neste estudo.

Os resultados dos programas de intervenção ainda são inconclusivos, entretanto têm se mostrado mais efetivos no que diz respeito à melhora das escolhas/hábitos alimentares e conhecimentos em nutrição/alimentação. Quanto à redução do IMC, dificilmente encontra-se resultados positivos, uma vez que, durante a infância, o desenvolvimento corporal é constante e em indivíduos saudáveis há aumento no peso e estatura, mesmo que discreto. Outro fator é a duração dos programas, geralmente curta para promover mudanças importantes e significativas.

O resultado do presente estudo foi positivo, principalmente no que diz respeito à prevenção do aumento da obesidade infantil e à redução do tempo de tela, o que sustenta a importância das intervenções de base escolar para a promoção de hábitos de vida saudáveis.

\section{Referências}

1. World Health Organization. World health statistics 2015. Geneva: WHO; 2015. Acesso em: 21 nov. 2015. $161 \mathrm{p}$.

2. Guimarães ACA, Feijó I, Soares A, Fernandes S, Machado Z, Parcias SR. Excesso de peso e obesidade em escolares: associação com fatores biopsicológicos, socioeconômicos e comportamentais. Arq Bras Endocrinol Metab. 2012; 56(2):142-148.

3. Margarey AM, Daniels LA, Boulton TJ, Cockington RA. Predicting obesity in early adulthood from childhood and parental obesity. Int J Obes Relat Metab Disord. 2003; 27(4):505-513.

4. Instituto Brasileiro de Geografia e Estatística. Pesquisa de Orçamentos Familiares 2008-2009: antropometria e estado nutricional de crianças, adolescentes e adultos no Brasil. Rio de Janeiro: IBGE; 2010. Disponível em: http://www.abeso.org.br/uploads/downloads/70/553a23f27da68.pdf 
5. Molina MCB, Faria CP, Montero MP, Cade NV, Mill JG. Fatores de risco cardiovascular em crianças de 7 a 10 anos de área urbana, Vitória, Espírito Santo, Brasil. Cad. Saúde Pública 2010; 26(5):909-917.

6. Andrade Neto F, Eto FN, Pereira TS, Carletti L, Molina MC. Active and sedentary behaviours in children aged 7 to 10 years old: the urban and rural contexts, Brazil. BMC Public Health 2014; 14:1174.

7. American Academy of Pediatrics. Committee on Public Education. Children, adolescents, and television. Pediatrics 2001; 107(2):423-26.

8. Rivera IR, Silva MAM, Silva RDTA, Oliveira BAV, Carvalho ACC. Atividade física, horas de assistência à TV e composição corporal em crianças e adolescentes. Arq Bras Cardiol. 2010; 95(2):159-165.

9. Hallal PC, Knuth AG, Cruz DKA, Mendes MI, Malta DC. Prática de atividade física em adolescentes brasileiros. Ciênc. Saúde Coletiva 2010; 15(2): 3035-42

10. Azevedo MR, Araújo CL, Silva MC, Hallal PC. Tracking of physical activity from adolescence to adulthood: a population-based study. Rev. Saúde Pública 2007; 41(1):69-75.

11. Gabriel CG, Santos MV, Vasconcelos FAG. Avaliação de um programa para promoção de hábitos alimentares saudáveis em escolares de Florianópolis, Santa Catarina, Brasil. Rev Bras Saúde Matern Infant. 2008; 8(3):299-308.

12. Vargas ICS, Sichieri R, Sandre PG, Veiga GV. Avaliação de programa de prevenção de obesidade em adolescentes de escolas públicas. Rev Saúde Pública 2011; 45(1):59-68.

13. World Health Organization. Obesity: preventing and managing the global epidemic. Report of a WHO Consultation. Geneva: WHO; 2000. (WHO technical report series, 894).

14. Fernandes CDR. Validação do questionário de avaliação da atividade física em crianças com idade entre 9 e 10 anos [dissertação]. Vitória: Universidade Federal do Espírito Santo; 2012.

15. Moser G. Psicologia ambiental e estudos pessoas-ambiente: que tipo de colaboração multidisciplinar? Psicol. USP 2005; 16(1-2):131-140.

16. Associação Brasileira de Empresas de Pesquisa Critério de classificação econômica Brasil [Internet]. [acesso em: 15 abr. 2015]. Disponível em: http://www.abep.org/criterio-brasil

17. World Health Organization. Growth reference data for 5-19 years [Internet]. Geneva: WHO; 2007. [Acesso em: 15 abr. 2015]. Disponível em: http://www.who.int/growthref/who2007_bmi_for_age/en/

18. Taylor RW, Jones IE, Williams SM, Goulding A. Evaluation of waist circumference, waist-to-hip ratio, and the conicity index as screening tools for high trunk fat mass, as measured by dual-energy X-ray absorptiometry, in children aged 3-19 y. Am J Clin Nutr. 2000; 72(2):490-5.

19. World Health Organization Global. Recommendations on physical activity for health [Internet]. Geneva: WHO; 2011. [acesso em: 21 abr. 2015]. Disponível em: http://www.who.int/dietphysicalactivity/ factsheet_recommendations/en/

20. Castilho SD, Nucci LB, Hansen LO, Assuino SR. Prevalência de excesso de peso conforme a faixa etária em alunos de escolas de Campinas, SP, Brasil. Rev Paul Pediatr. 2014; 32(2):200-206.

21. Rosaneli CF, Auler F, Manfrinato CB, Rosaneli CF, Sganzerla C, Bonatto MG et al. Avaliação da prevalência e de determinantes nutricionais e sociais do excesso de peso em uma população de escolares: análise transversal em 5.037 crianças. Rev Assoc Med Bras. 2012; 58(4):472-476. 
22. Leal VS, Lira PIC, Oliveira JS, Menezes RCE, Sequeira LAS, Arruda NMA et al. Excesso de peso em crianças e adolescentes no Estado de Pernambuco, Brasil: prevalência e determinantes. Cad Saúde Pública 2012; 28(6):1175-1182.

23. Ribas AS, Silva LCS. Fatores de risco cardiovascular e fatores associados em escolares do Município de Belém, Pará, Brasil. Cad Saúde Pública 2014; 30(3):577-586.

24. Brasil LMP, Fisberg M, Sousa MH. Excesso de peso de escolares em região do Nordeste Brasileiro: contraste entre as redes de ensino pública e privada. Rev Bras Saúde Matern Infant. 2007; 7(4):405-412.

25. Molina MCB, Lopéz PM, Faria CP, Cade NV, Zandonade E. Preditores socioeconômicos da qualidade da alimentação de crianças. Rev Saúde Pública 2010; 44(5): 785-92.

26. Benzecry SG, Mello ED, Escrivão MAMS. Alimentação do escolar. In: Sociedade Brasileira de Pediatria Manual de orientação: alimentação do lactente, alimentação do pré-escolar, alimentação do escolar, alimentação do adolescente, alimentação na escola. São Paulo: Departamento de Nutrologia; 2006. 64 p.

27. Coelho LG, Cândido AP, Machado-Coelho GL, Freitas SN. Association between nutritional status, food habits and physical activity level in schoolchildren. J Pediatr. (Rio J) 2012; 88(5):406-412.

28. Vasconcellos MB, Anjos LA, Vasconcellos MTL. Estado nutricional e tempo de tela de escolares da Rede Pública de Ensino Fundamental de Niterói, Rio de Janeiro Brasil. Cad. Saúde Pública 2013; 29(4):713-722.

29. Oliveira TC, Silva AAM, Santos CJN, Silva JS, Conceição SIO. Atividade física e sedentarismo em escolares da rede pública e privada de ensino em São Luís. Rev. Saúde Pública 2010; 44(6):996-1004.

30. Pearson N, Braithwaite RE, Biddle SJ, van Sluijs EM, Atkin AJ. Associations between sedentary behaviour and physical activity in children and adolescents: a meta-analysis. Obes Rev. 2014; 15(8):666-75.

31. Maddison R, Marsh S, Foley L, Epstein LH, Olds T, Dewes O, et al. Screen- Time Weight-loss Intervention Targeting Children at Home (SWITCH): a randomized controlled trial. Int J Behav Nutr Phys Act 2014, 11:111.

32. Lopes L, Lopes VP, Pereira B. Atividade física no recreio escolar: estudo de intervenção em crianças dos seis aos 12 anos. Rev Bras Educ Fís Esp. 2006; 20(4):271-80.

33. Ribeiro, RQC, Alves L. Comparison of two school-based programmes for health behaviour change: the Belo Horizonte Heart Study randomized trial. Public Health Nutr. 2014; 17(6):1195-204.

34. Costa AGM, Gonçalves AR, Suart DA, Suda G, Piernas P, Lourena LR, et al. Avaliação da influência da educação nutricional no hábito alimentar de crianças. Rev Inst Ciênc Saúde 2009; 27(3):237-43.

Recebido: 15/3/2016

Aceito: 25/8/2016 
